\title{
Summary Talk on Cosmology and Gravitation XXII Brazilian National Meeting on Particles and Fields
}

\author{
M.J. Rebouças \\ Centro Brasileiro de Pesquisas Fúsicas \\ Departamento de Relatividade e Partículas \\ Rua Dr. Xavier Sigaud 150, 22290-180, Rio de Janeiro, RJ, Brazil
}

Received on 29 March, 2002

\begin{abstract}
The plenary lectures, parallel talks, oral presentations and poster contributions on Cosmology and Gravitation presented during the XXII Brazilian National Meeting on Particles and Fields are briefly reviewed. Some general remarks are also presented.
\end{abstract}

\section{Overview and introduction}

After four days of listening to two plenary lectures, two parallel talks, a general public lecture, seventeen oral presentations, and reading more than fifty posters related to Cosmology and Gravitation, my chief impression is that the XXII Brazilian National Meeting on Particles and Fields (XXII BNMPF) was a noticeable scientific meeting, which covered a great variety of issues on Gravitation and Cosmology. It brought together perhaps most of the researchers and graduate students that have worked in this area in Brazilian Universities and Research Institutes. The sessions were very well attended with a fair number of clarifying questions. In general the speakers explained the underlying ground, approaches and results in a clear manner and kept to the time limits. I thank to them all for their quite good talks.

From this and the previous meetings I support the view that if one considers the contributions presented in, e.g., two successive BNMPF meetings they are rather representative of the researches that have been done in this area in Brazil, and most of the contributions reveal a clear emphasis on theoretical and (or) mathematical aspects of Gravitation and Cosmology [1].

The researches in modern cosmology can be broadly grouped in five domains:

i. Astrophysical cosmology;

ii. Observational cosmology;

iii. The early universe;

iv. Theoretical and mathematical cosmology; v. Cosmic topology (more recently).

Most of the contributions to the XXII BNMPF (organized by the Brazilian Physical Society - SBF) were in the last three families. On the other hand, as far as I am aware a great number of the observationallyoriented works on cosmology are often presented in the annual meeting of the Brazilian Astronomical Society (SAB).

I do not share the view that theoretical (and mathematical) cosmology should be strictly related to the observed universe, otherwise it becomes a pointless exercise. In my evaluation the frontier between theoretical (and mathematical) cosmology and more observationally-oriented cosmology is not and should not be well-defined, and it is very important that we have people working on both general aspects of modern cosmology. However, I feel that a closer interaction between theoretical and observationally-oriented cosmologists is more than desirable - in my view it is recommendable - for the benefit of both (physicist and astophysicist) communities. The recent workshop called New Physics in the Space is certainly a good example in this direction [2].

A considerable number of abstracts were submitted to the organizers. I envisage how hard was to select between oral and poster presentations. A number of relevant subjects seemed to them to be of interest to motivate oral presentations, but no doubt other organizers would have made a quite different, and perhaps equally good, selection. I will summarize the presented contributions below, with apologies to their authors for any unintentional misapprehension and (or) misrepresentation. 


\section{Plenary and parallel talks}

The General Relativity (GR) predicts the existence of gravitational waves, and analyses of experimental data obtained from observations of binary star systems seem to give a clear evidence that these systems loose energy through gravitational radiation [3].

Gravitational waves are predicted not only by GR but also by other metric gravitational theories of some generality and scope. However, the characteristics of gravitational waves such as propagation speed, multipolar structures and polarization states vary from one theory to another. Thus, it is expected that its detection will provide a rather relevant tool to select the most suitable theory of gravitation [4]. Further, the direct observation of gravitational waves also offers the possibility of testing GR (as well as other metric theories) in a strong field limit, where the effects are not merely a correction of Newtonian Gravitation. Obviously at Earth the waves are expected to be weak perturbations, however they can in principle provide information on the conditions at their strong field sources [5]. Further, the technology needed to detect as well as the analysis of gravitational waves is expected to open a new window for observation of the universe, thus giving rise to a remarkable period in astronomy and cosmology.

Barry C. Barish (California Institute of Technology) delivered a clear plenary lecture in the XXII BNMPF, in which he presented a good review of the new generation of detectors with suitable sensitivities for detecting gravitational waves from a variety of catastrophic events, such as the gravitational collapse of stars or coalescence of compact binary systems. He pointed out that more than forty years after the beginning of the search for gravitational waves, several resonant-mass wave detectors are monitoring the most strong potential sources of such waves (in the $\approx k \mathrm{~Hz}$ band) in our galaxy and in our local group. The gravitational wave detection of low frequencies (in the $\approx m \mathrm{~Hz}$ band) from space was also briefly discussed in his talk. Contrarily to the earth based detectors, for this type of detection there is a natural advantage which is the fact that essentially all the noises due to the ground are eliminated (for more detail see [6]).

For some decades since the discovery of the cosmic expansion by Hubble [7] it was taken for granted that the matter content of the universe was composed of the forms of energy we can readily detect, i.e., the ordinary matter and radiation. Such universe would expand with a negative expansion rate due to gravitational self-attraction of the matter. However, some years later, theoretical aspects of the stability of galaxies and some observations (motion of cluster of galax- ies and stars and surrounding galaxies) indicated that most of the matter in the universe does not emit or absorb light (dark matter). The dark matter clear indication found resonance in the 1980's by the inflationary universe scenario in which the universe is taken to be flat - the total energy density equal to the critical density $\rho_{c}=3 H_{0}^{2} / 8 \pi G \approx 1.710^{-29} \mathrm{~g} / \mathrm{cm}^{3}$. However, measurements made by that time offered a clear indication that the ordinary matter and the radiation could only account for about $10 \%$ of this value - inflation seemed to require dark matter. The new consensus model became that in which the universe contains primarily cold, nonbaryonic dark matter, and although observations at that time indicated that the total mass density was smaller than the critical density, many cosmologists assumed that the flat model was reasonably well-established. However, further observations strongly indicated that the total matter density was indeed less than half of the critical density. The only way of having such a low mass density and a flat universe is if an additional, nonluminous and nonclustering "dark energy" component dominates the universe today, although it must have been negligible in the past so as to permit structure formation. The general relativistic Friedmann-Lemaitre-Robertson-Walker (FLRW) standard modeling approach requires that this dark energy has a negative pressure, and the recent measurements of distant exploding stars (supernovae) support the existence of a negative-pressure dark energy such that the (total) energy density $\rho$ and the (total) pressure $p$ obey the relation $\rho+3 p<0$, which leads to an accelerating expansion for the universe today (for a fair list of basic references on the topics I have briefly mentioned here, see the very good review by Bahcall et al. [8]).

Luca Amendola (Osservatorio Astronomico di Roma) presented a very good plenary talk in which he reviewed how the recent independent cosmological observations constrain the values of the cosmological density parameters, and have indicated that the universe is filled with dark matter and dark energy and undergoes an accelerating expansion. He pointed out how recent supernovae Ia observations $[9,10]$ call for an acceleration expansion of the universe, which together with recent cosmic microwave, large-scale structure and lensing data give a rather strong indication that the matter (energy) content of the universe is basically composed of dark matter $(\sim 30 \%)$, dark energy $(\sim 70 \%)$, baryonic matter (very few percent). For more details and references see his review paper in this issue [11].

Odylio D. Aguiar (INPE) told us about the status of the Mario Schenberg gravitation wave detector, which is being constructed in the Institute of Physics of the University of São Paulo, and is expected to start to op- 
erate in 2004. This detector has a spherical antenna, will operate at temperature of less than $0.1 K$, and will be sensitive to signals of amplitude $h>10^{-21} \mathrm{~Hz}^{-1 / 2}$ in the bandwidth $3.0-3.4 k H z$ (for more details on this talk see Aguiar's paper in this issue [12, 13]).

It is well known that general relativity is a local metrical theory and therefore its field equations do not fix the topology of the spacetime, since geometry does not in general dictate the topology. This freedom has resulted in a great deal of recent works in which the possibility that the universe may possess a spatial section with a non-trivial topology is examined. Whether we live in a finite or infinite space and what is the size and the shape of the universe are open problems of topological nature. In his talk Helio V. Fagundes presented a brief introduction to cosmic topology in the context of FLRW models, and reviewed the work done by his group and collaborators at IFT/UNESP. The works he described range from early attempts to solve a controversy about quasars through the multiple images produced by nontrivial topologies, to more recent issues such as cosmic crystallography and quantum cosmology in compact universe models (for more details on this talk see Fagundes' paper in this issue [14]).

Before reviewing the oral presentations I remark that one particular objective of the organizers was to involve as far as possible the local general public with the meeting. To this end they invited I. Waga to give a public general lecture on cosmology, and he presented a clear and motivating lecture on the expansion of the universe [15].

\section{Oral presentations I}

The first oral presentation was of an observationallyoriented paper by M.O. Calvão, J.R.T. de Mello Neto and I. Waga [16], where it was discussed, through Monte Carlo simulations, how the Alcock-Paczyński test, as applied to quasar clustering, can be used to probe the cosmological density parameters space, and also the equation of state parameter. The approach discussed in this contribution may become a relevant strategy to narrow down the cosmological parameter space.

H.P. de Oliveira and S.E. Jorás presented an early universe contribution, where they studied the evolution of the entropy and adiabatic perturbations and showed that for a small amount of dissipation the entropy perturbations can be neglected, and so the purely adiabatic perturbations will be responsible for the primordial spectrum of inhomogeneities [17].

In a work presented by Sandra Roveda (M.E.
Araújo, S.R.M.M. Roveda and W. Stoeger [18]) spherically symmetric perturbations of FLRW models in the so-called observational coordinates were examined. The FLRW (spherical) models are used as the background spacetime and a particular case of spherical deviation from this background is studied (for more details on this "observational program" I refer the reader to the paper by Ellis et al. [19]).

Using recent observational constraints on cosmological density parameters, together with recent mathematical results concerning small volume hyperbolic manifolds and a topological detectability indicator introduced in [20], G.I. Gomero reported on a contribution on cosmic topology in which it is argued that, by employing pattern repetitions, the topology of nearly flat small hyperbolic universes can be observationally undetectable [21]. This is an important result in view of the fact that quantum cosmology seems to favour universes with small volumes, and from the expectation, coming from inflationary scenarios, that the total density $\Omega_{0}$ is likely to be very close to one.

By considering that the current expanding era is preceded by a contracting phase, in other words by assuming the existence of one bounce, N. PintoNeto showed how one can conclude that no observable bounce could possibly have taken place in the early universe if GR together with hydrodynamical fluids describe the evolution of the universe, thus under these conditions the universe has always expanded (see [22] for more details).

The first set of oral presentation was closed with the presentation by S.E. Jorás of a article by R.H. Brandenberger, S.E. Jorás and J. Martin, in which the spectrum of scalar field fluctuations in a bouncing asymptotically flat universe was calculated, and the dependence of this result on length scales shorter than the Planck length was investigated (for more details see [23]).

\section{Oral presentations II}

Topological defects such as monopoles, strings and domain walls have been studied in different contexts such as to understand the primordial universe and structure formation in the early universe. Their nature depends upon the topology of the vacuum manifold of the field theory under consideration. The second set of oral presentations began with a contribution by R.M. Teixeira Filho and V.B. Barbosa [24], where they have obtained in the context of scalar tensor theories the gravitational field of a global monopole, in the weak-field approximation, extending the Barriola and Vilenkin monopole solution found in the context of GR. 
S.S. e Costa delivered a contribution related to cosmic topology in which general solutions of the Helmholtz equation, in several coordinates systems for two and three-dimensional hyperbolic, spaces were presented [25].

Another contribution related to cosmic topology was an article by D. Müller, H.V. Fagundes and R. Opher [27], where they have studied, through numerical calculations, the Casimir (vacuum) energy for a conformally coupled, massive scalar field in a static universe whose spatial sections are endowed with the topology of the smallest known hyperbolic three-manifold (Weeks manifold). They have concluded that there is a spontaneous vacuum excitation of low multipolar components.

A. Bernui also presented a cosmic topology contribution in which the cosmic crystallography statistical approach to the topological signature of the universe was used in his numerical simulations with incomplete catalogs.

R. Androvandi presented an interesting contribution (R. Aldrovandi, J. Gariel and G. Marcilhacy) on what they call the pre-nucleosynthesis period (PNS period), between $z \approx 10^{10}$ and $z \approx 10^{15}$, and where our local physics does not necessarily hold. A general overview of physical problems appearing in the PNS period was given, and possible understanding for the striking outcomes were discussed. For more details see [26].

\section{Oral presentations III}

Condensed matter systems such as light in moving dielectrics and quasiparticles in a moving superfluid can be used to mimic kinematic aspects of general relativity. The comparative study between the kinematical aspects of GR and other kinds of interactions has been called analog model for gravitation or simply analog gravity. These types of analog models are rather important in that, e.g., they provide black hole analogs and may lead to experimental test of quantum field theory in curved space. The last set of oral presentations began with a brief review talk, delivered by R. Klippert (a work in collaboration with V.A. de Lorenci) where analog gravity models were discussed in several contexts, ranging from electrodynamic in non-linear media [28] to moving dielectrics and acoustic perturbations (for a fair number of references on this topic see [29]).

A work by J.M.F Maia and J.A.S. Lima in which a procedure to generate cosmological solutions in the context of GR whose matter content is given by a scalar field plus a perfect fluid was presented. Applications to inflationary and quintessence cosmologies were also considered in specific models (see [30]).
A.Y. Miguelote reported on the results of an article in collaboration with M.F.A. Silva, A.A. Wang and N.O. Santos, in which some properties of the Levi-Civita (LC) type of solutions of coupled EinsteinMaxwell equations are studied, and some limits of this type of solution are obtained together with an interpretation of the free parameters involved in LC type of spacetimes [31].

I.D. Soares presented a interesting paper (homoclinic chaos in the dynamics of a general Bianchi IX) of a work in collaboration with H.P. Oliveira, A.M.O. Almeida, and E.V. Tonini, in which they have studied the dynamics of anisotropic (three scale factors) Bianchi type IX model with dust and cosmological constant (positive). They have examined the existence of chaos in this model, and have shown that it is chaotic, and that the chaos has a homoclinic nature. The role played by the cosmological constant in the phase space is shown to be crucial in that it determines both the existence of a saddle-center-center critical point, and the critical points at infinity corresponding to the DeSitter configuration. For more details I refer the reader to ref. [32].

The interaction of Hawking radiation and a static electric charge was a contribution delivered by L.C.B. Crispino of a work in collaboration with A. Higuchi and G.E.A. Matsas, in which they have investigated in two interacting situations whether the equality found for the response of a static scalar field is maintained in the case of electric charges (see [33]).

The last oral presentation was by J. Castiñeiras (a work in collaboration with L.C.B Crispino, G.E.A. Matsas and D.A.T. Vanzella). They have studied "free particles" for which $E<m c^{2}$ outside ReissnerNordstrom blackholes and in a spacetime of a star (for more details see [34]).

\section{Panel contributions}

More than fifty contributions were presented as posters. About twenty per cent were related to the physics of the early universe. F.G. Alvarenga, J.C. Fabris, G.A. Monerat and N.A. Lemos presented two contributions on quantum cosmology [35]. N. Pinto-Neto and E.S. Santini also presented a poster related to quantum cosmology (see in this connection the article [36]). Three works connected to the preheating process were presented as posters: one by S.E. Jorás and V.H. Cárdenas [37]; a second by A.H. Campos, R. Rosenfeld and J.M.F. Maia; and another by R.O. Ramos and S.E. Jorás. Three posters were related to inflation one by H.P. de Oliveira and I. Damião Soares, 
a second by H.P. de Oliveira alone [38], and a third by J.C. Fabris, A.M. Pelinson and I.L. Shapiro [39]. A.H. Campos, R. Rosenfeld, J.M.F. Maia and H. Reis discussed in a poster the possible production of superheavy primordial particles. R. Aldrovandi, A.L. Barbosa, M. Cançada and J.G. Pereira presented a poster on kinematics of a spacetime with infinite cosmological constant $(\Lambda \rightarrow \infty)$ and speed of light $(c \rightarrow \infty)$, whose results may be of some interest to early universe cosmology [40]. A poster with some contact with the early universe was also brought to the meeting by M.G.C. Batista.

Nine contributions related to topological defects in different contexts were presented as posters. Three of them treated these defects in scalar-tensor theories: one by V.B. Bezerra, L.P. Colatto, M.E.X. Guimarães and R.M. Teixeira Filho [41]; another by V.B. Bezerra and C.N. Ferreira [42]; and a third by V.B. Bezerra, R.M. Teixeira Filho, G. Grebot and M.E.X. Guimarães [43]. The behaviour of nonrelativistic quantum particles interacting with different potentials in the spacetimes generated by a cosmic string and also by a global monopole were discussed in a poster by G. de A. Marques and V.B. Bezerra [44]. Two posters on cosmic strings were presented: one by A.A. Morégula in collaboration with M.M. Som, and another by V.A. de Lorenci and E.S. Moreira Jr (see [45]). The solutions of Schrödinger equations for the Coulomb and harmonic oscillator potential in the cosmic-string conical spacetimes of $N$ dimensions were presented in a poster by J.L.A. Coelho and R.L.P.G. Amaral [46]. Topological defects in the context of (or related to) condensed matter were discussed in two posters: one by C.A.L. Ribeiro, C. Furtado and F. Moraes, and another by J.F de Assis, V.B. Bezerra and C. Furtado.

Besides Fagundes' parallel talk and the oral presentations by G.I. Gomero, S.S. e Costa, D. Müller et al., and A. Bernui, we had an additional poster presentation by G.I. Gomero of a work in collaboration with M.J. Rebouças and R. Tavakol [20] on the detectability of cosmic topology of nearly flat FLRW universes in the light of the most recent observations (Sne Ia, large scale structure (LSS) observations, MAXIMA and BOOMERANG).

In addition to Barry C. Barish's plenary talk, and O.D. Aguiar's parallel talk, four posters related to gravitational waves were presented: K.L. Ribeiro and O.D. Aguiar; S.R. Furtado and O.D. Aguiar; and L.A. de Andrade, O.D. Aguiar and K.L. Ribeiro; and also C. Frajuca, N.S. Magalhães, O.D. Aguiar, K.L. Ribeiro, L.A. de Andrade, W.F. Velloso Jr and J.L. Melo (see [47, 48]).

Three posters were presented on parallel gravity.
One by T. Vargas on Regge Calculus in teleparallel gravity, and three others by A.A. Sousa and J.W. Maluf (for more details see $[49,50]$ ).

It seems indisputable that most of theoretical physicists spend a large amount of their time in carrying out nonnumeric calculation of one sort or another, most of which are algorithmic or semi-algorithmic. Today the computer algebra (CA) systems are standard tools of theoretical physicists for performing symbolic, although in most cases perhaps they simply do not mention this fact. We had three CA poster contributions in the meeting, all related to the Maple system. One dealt with the symmetry groups in GR (by J.A.F. Roveda and J.E.F. Skea), which is a relevant piece of information needed in the invariant (local) characterization of spacetime; and another was about the usage of Maple to calculate quasi-normal modes of radiating systems (by P.C.C. Monteiro Jr and J.E.F. Skea). F.D. Sasse delivered a contribution in collaboration with R.F. McLenaghan and S.R. Czapor where the Maple package NPspinor was used to prove the Hadamard's conjecture for the scalar wave equation in Petrov type III backgrounds.

Different aspects and contexts of black holes were discussed in a series of four posters. G. de Oliveira Neto studied exact solutions of Einstein's equations in $(2+1)$-dimensions which, depending on the values of certain parameters, may represent the formation of black holes [51]. A.A. Sobreira and V.B. Bezerra examined rotating black hole in Einstein-Fokker theory in $(2+1)$-dimensions. Holonomies in the $(2+1)$ dimensional geometry of black holes were the content of a poster by A. Carvalho, C. Furtado and F. Moraes. A black hole type solution of Einstein equations in 2dimensions was discussed by D.G. Delfrate.

There were four posters which I broadly grouped as gravitational-related works. M. Calçada presented a poster on gravitation and the local symmetry group of spacetime [52]. A.A. Sousa and J.W. Maluf presented a poster where they proposed an experimental test for the gravito-magnetic effect. R.C. Rigitano discussed in an interesting poster the geometric representation of general linear transformations, and the possibilities and limitations of the representation of covariant transformations. C.R. Muniz examined geometric properties of the spin in the context of GR.

Two posters were related to Astrophysics. In one the third post-Newtonian dynamics of compact binaries equations of motion was discussed by V. Andrade, L. Blanchet and G. Faye (for detail see [53]). In another by K. Sau Fa and I.T. Pedron it is used Tsallis' distribution functions to examine the galaxies in equi- 
librium configurations with spherical symmetry (King models) [54].

M.D. Maia presented an interesting poster (related to two works in collaboration with E.M. Monte $[55,56]$ ) in which he showed how brane-world cosmology offers a quite simple explanation for dark energy.

M.E.X. Guimarães, L.P. Colatto and F.B. Tourinho presented a poster in which they extended to the context of scalar-tensor theories [57] (where the parameter $\omega$ in no longer constant) a procedure, devised by A. Barros and C. Romero [58], which makes clear how to obtain solutions in the Brans-Dicke theory from solutions in GR for the same matter content when one considers the weak-field approximations in both theories.

In an interesting poster by S.O. Mendes and R. Opher it was discussed how the $1 / r^{2}$ gravitational law on submillimetric scale can be used to test the modified Newtonian dynamic theory (MOND) [59].

Besides the brief review talk, presented by R. Klippert as an oral presentation, Analog Gravity also appeared in a poster by V.A. De Lorenci and R. Klippert [28].

An interesting poster was presented by F.P. Devecchi and G. Kremer in which the kinetic theory of relativistic gases in two-dimensional space was developed to obtain thermodynamical quantities in 2-dimensional cosmological models [60].

H.P. de Oliveira and I. Damião Soares presented in a contribution the results of their studies of the dynamics of spherically symmetric gravitational collapse of a massless scalar field [61]. H. P. de Oliveira, I. Damião Soares and E.V. Tonini also had a poster where they presented an analytical method to describe the unstable periodic orbits of the center manifold for Hamiltonian systems. Another poster with some relation to this was presented by O.C. Castellani, G.A. Monerat and J.F.V. Rocha.

M.M. de Souza claimed in a poster that the constant anomalous radial acceleration observed in the Pioneer 10 and 11 spacecrafts can be explained in terms of discrete interactions (for detail see [62, 63]).

J.F. Villas da Rocha presented a poster in which solutions to Einstein's field equations in $N$-dimensions with spherical symmetry were discussed [64].

L. Sandoval Jr considered in a contribution the constraints on generalized metrics (with both symmetric and antisymmetric parts) described through vierbeins imposed by bosonic strings in curved spacetimes.

M.L. Bedran presented an interesting pedagogical poster comparing the Doppler (special relativity) effects with the cosmological redshift of general relativity.

F.D. Sasse reviewed the different interpretations of the Mashhoon effect, and discussed a description of fermions in a storage ring in a Serret-Frenet tetrad frame, instead of the usual Hehl-Ni frame.

M.J. Lazo and S. Ragusa showed how to calculate the electromagnetic angular momentum emission of fourth order, extending therefore the calculations made in the Landau-Lifchitz book for electric dipole. They intend to use a similar scheme to examine analogous gravitational emission.

To close this section I remark that a considerable number of extended versions of the works presented in the XXII BNMPF will be available (proceedings of the meeting) and can fairly soon be obtained via download from the Brazilian Physical Society (SBF) webpage. So, for more details on some of works briefly reviewed here, I refer the reader to that site [65].

\section{Closing remarks}

The large number of abstracts submitted to the Cosmology and Gravitation sessions of the XXII BNMPF is certainly indicative of the robust level of interest in the area. The meeting was a lively one and demonstrated the vitality of a extensive set of research topics. It also was very informative and many discussions took place in the regular sessions, and also in the coffee and others breaks. The spirit of collaboration, essential to the progress of science, was present in the sessions and discussions.

Let me close my review by strengthening a point I have indicated in the introduction, which is that the interaction and collaboration between theoretical and observationally-oriented gravito-cosmologists is strongly advisable for the advance of the area, the benefit of our community as well as for the formation of Brazilian young scientists in Cosmology and Gravitation.

\section{Acknowledgments}

I would like to thank the Organizing Committee for the invitation to deliver the summary talk on Gravitation and Cosmology of the XXII BNMPF. I also thank them for the large amounts of effort put into the organization of a very successful meeting. I thank Ioav Waga for his help in the classification of the topics for my summary talk. Some authors provided me with preprints, reprints or references related to their submitted abstracts. The additional information material proved to be very useful for me, and I thank all these colleagues for their helpful gesture. I also thank Antonio F.F. Teixeira and G.I. Gomero for reading the manuscript and for the useful suggestions. Finally, I 
thank $\mathrm{CNPq}$ for the grant under which this work was carried out.

\section{References}

[1] I. Waga, Braz. J. Phys. 31, 285 (2001).

[2] See the of abstracts of contributed papers, Workshop: New Physics in the Space, held in Campos do Jordão, March 03 to 08,2002 , and organized by R. Opher (IAG/USP), C. Escobar (IF/UNICAMP), G. Matsas (IFT/UNESP), O. Aguiar (DAS/INPE), P. Pellegrini (ON), R. Rosenfeld(IFT/UNESP), S.M. Viegas (IAG/USP), T. Villela (DAS/INPE) and Z. Abraham (IAG/USP)

[3] J.H. Taylor, Rev. of Mod. Phys. 66, 711 (1994). See also references therein.

[4] C.M. Will, Theory and Experiment in Gravitational Physics. Cambridge University Press (1981).

[5] B.C. Barish, Gravitational Waves the New Generations of Laser Interferometric Detectors, in "Proceedings of the Ninth Marcel Grossmann Meeting on General Relativity (2000), V. Gurzadyan, R.T. Jantzen, R. Ruffini, Eds., World Scientific, Singapore, 2002. Available at http://www.icra.it/MG/mg9/mg9.htm

[6] B.C. Barish, Braz. J. Phys., 32, 832 (2002).

[7] E. Hubble, Proc. Nat. Acad. Sci. 15, 168 (1929).

[8] N.A. Bahcall, J.P. Ostriker, S. Perlmutter and P.J. Steinhardt, Science 284, 1481 (1999).

[9] S. Perlmutter et al., Astrophys. J. 517, 565 (1999).

[10] A.G. Riess et al., Astron. J. 116, 1009 (1998).

[11] L. Amendola, et al., Braz. J. Phys., 32, 823 (2002).

[12] O.D. Aguiar, Braz. J. Phys., 32, 866 (2002).

[13] O.D. Aguiar et al., Class. Quantum Grav. 19, 1949 (2002).

[14] H.V. Fagundes, Braz. J. Phys., 32, 891 (2002). Also preprint gr-qc/0112078 (2001).

[15] I. Waga, the text of this lecture is available at http://www.if.ufrj.br/ ioav/nota.html.

[16] M.O. Calvão, J.R.T. Mello Neto and I. Waga, Phys. Rev. Lett. 88, 091302 (2002).

[17] H.P. Oliveira and S.E. Jorás, Phys. Rev. D 64, 063513 (2001).

[18] M.E. Araújo, S.R.M.M. Roveda and W. Stoeger, "Perturbed Spherically Symmetric Dust Solution of the Field Equations in Observational Coordinates with Cosmological Data Functions", preprint gr-qc/0105001 (2001).

[19] G.F.R. Ellis, S.D. Nel, R. Maartens, W.R. Soeger and A.P. Whitman, Phys. Rep. 124315 (1985).

[20] G.I. Gomero, M.J. Rebouças and R. Tavakol, Class. Quantum Grav. 18, 4461 (2001).

[21] G.I. Gomero, M.J. Rebouças and R. Tavakol, Class. Quantum Grav. 18, L145 (2001).
[22] P. Peter, N. Pinto-Neto, Phys. Rev. D 65, 023513 (2002).

[23] R.H. Brandenberger, S.E. Jorás, J. Martin, "TransPlanckian Physics and the Spectrum of Fluctuations in a Bouncing Universe", preprint hep-th/0112122 (2001).

[24] R.M. Teixeira Filho and V.B. Bezerra, Phys. Rev. D 64067502 (2001).

[25] S.S. e Costa, "A Description of Several Coordinate Systems for Hyperbolic Spaces", preprint mathph/0112039 (2001).

[26] R. Aldrovandi, J. Gariel and G. Marcilhacy, "On the Pre-nucleosynthesis Cosmological Period", preprint grqc/0203079 (2002)

[27] D. Müller, H.V. Fagundes and R. Opher, Phys. Rev. D 63, 123508 (2001).

[28] V.A. De Lorenci and R. Klipert, Phys. Rev. D 65, 064027 (2002).

[29] See the workshop web page on analog models of general relativity at http://www.cbpf.br/ bscg/analog

[30] J.M.F Maia and J.A.S. Lima, Phys. Rev. D 63, 083513 (2002).

[31] A.Y. Miguelote, M.F.A. da Silva, A. Wang, and N.O. Santos, Class. Quant. Grav. 18, 4569 (2001).

[32] H.P. de Oliveira, A.M. Ozório de Almeida, and I. Damião Soares, E.V. Tonini, "Homoclinic Chaos in the Dynamics of a General Bianchi IX Model", preprint grqc/0202047(2002), to appear in Phys. Rev. D (2002).

[33] L.C.B. Crispino, A. Higuchi and G.E.A. Matsas, Phys. Rev. D 58, 084027 (1998).

[34] J. Castiñeiras, L.C.B. Crispino, G.E.A. Matsas and D.A.T. Vanzella, "Free Massive Particles with Total Energy $E<m c^{2}$ in Curved Spacetimes", preprint grqc/0201093 (2002).

[35] F.G. Alvarenga, J.C. Fabris, N.A. Lemos and G.A. Monerat, "Quantum Cosmological Perfect Fluid Models", preprint gr-qc/0106051. To appear in Gen. Rel. Grav. (2002).

[36] J.A. de Barros, N. Pinto-Neto and M.A. Sagioro-Leal Phys. Lett. A 241, 229 (1998).

[37] S.E. Jorás and V.H. Cárdena, "Chaos and Preheating", preprint gr-qc/0108088 (2001).

[38] H.P. de Oliveira, Phys. Lett. B 526, 1 (2002).

[39] J.C. Fabris, A.M. Pelinson and I.L. Shapiro, Nucl. Phys. B 597, 539 (2001); Erratum-ibid. 602, 644 (2001).

[40] R. Aldrovandi, A.L. Barbosa, M. Cançada and J.G. Pereira, "Kinematics of a Spacetime with Infinite $\Lambda$ and $c "$, preprint gr-qc/0105068 (2001).

[41] V.B. Bezerra, L.P. Colatto, M.E.X. Guimarães and R.M. Muniz Filho, "Scalar and Spinor Particles in the Spacetime of a Domain Wall in String Theory", Phys. Rev. D 65, in press (2002), also preprint gr-qc/0104038 (2001).

[42] V.B. Bezerra and C.N. Ferreira, Phys. Rev. D 65, in press (2002), also preprint hep-th/0111167 (2001). 
[43] V.B. Bezerra, R.M. Teixeira Filho, G. Grebot and M.E.X. Guimarães, Mod. Phys. Lett. A 16, 1565 (2001).

[44] G. de A. Marques and V.B. Bezerra, Class. Quantum Grav. 19, 985 (2002).

[45] V.A. De Lorenci and E.S. Moreira Jr, Phys. Rev. D 63, 027502 (2001).

[46] J.L.A. Coelho and R.L.P.G. Amaral, "Coulomb and Quantum Oscillator Problems in Conical Spaces with Arbitrary Dimensions", preprint gr-qc/0111114 (2001).

[47] K.L. Ribeiro et al., Class. Quantum Grav. 19, 1967 (2002).

[48] C. Frajuca et al. Class. Quantum Grav. 19, 1961 (2002).

[49] A.A. Sousa and J.W. Maluf, Prog. Theor. Phys. 104, $531(2000)$

[50] J.W. Maluf and A.A. Sousa, "Hamiltonian Formulation of Teleparallel Theories of Gravity in the Time Gauge", preprint gr-qc/0002060 (2000).

[51] G. de Oliveira Neto, "Self-similar Collapse of a Massless Scalar Field in Three-dimensions", preprint grqc/0105077 (2001).

[52] M. Calçada, Int. J. Theor. Phys. 41, 729 (2002).

[53] V. Andrade, L. Blanchet and G. Faye, Class. Quant. Grav. 18, 753 (2001).

[54] K. Sau Fa and I.T. Pedron, "Extended King Models for Star Clusters", preprint astro-ph/0108370.
[55] M.D. Maia, E.M. Monte, "Geometirc Stability of Brane-worlds", preprint hep-th/0103060 (2001).

[56] M.D. Maia, E.M. Monte, "Geometry of Brane-Worlds", preprint hep-th/0110088 (2001).

[57] M.E.X. Guimarães, L.P. Colatto and F.B. Tourinho, Spacetime and Substance 2, 71 (2001).

[58] A. Barros and C. Romero, Phys. Lett. A 245, 31 (1998).

[59] S.O. Mendes and R. Opher, Phys. Lett. B 522, 1 (2001).

[60] G.M. Kremer and F.P. Devecchi, "Thermodynamics and Kinetic Theory of Relativistic Gases in 2-D Cosmological Models", to appear in Phys. Rev. D 65 (2002), also preprint gr-qc/0202025 (2002).

[61] H.P. de Oliveira and I. Damião Soares, Phys. Rev. D 65, 064029 (2002).

[62] M.M. de Souza, "Gravity and Antigravity with Discrete Interactions: Anternatives I and II", preprint grqc/0111052 (2001).

[63] M.M. de Souza, "Discrete Interactions and the Pioneer Anomalous Acceleration: Alternative II", preprint grqc/0106047 (2001).

[64] J.F.V. Rocha, Int. J. Mod. Phys. D 11, 113 (2002).

[65] See Brazilian Physical Society (SBF) web page at http://www.sbf.if.usp.br 